\title{
Effects of Mutation and Random Drift on Leonard's Gene-for-Gene Coevolution Model
}

\author{
G. C. Kirby and J. J. Burdon
}

First author: School of Biological Sciences, Flinders University, GPO Box 2100, Adelaide, SA 5001, Australia; and second author: Centre for Plant Biodiversity Research, Division of Plant Industry, CSIRO, GPO Box 1600, Canberra, ACT 2601, Australia. Accepted for publication 15 January 1997.

For some time, controversy has surrounded the nature of the equilibrium properties of population genetics models of gene-forgene interactions between plants and pathogens (10). Even simple population genetics models contain considerable complexity, and recent ecological models (4) are often only superficially understood. The starting point for all these models is the gene-for-gene interaction recognized in many plant-pathogen interactions (16). In the simplest case, this consists of a host locus and a corresponding pathogen locus each with two alleles (designated 'resistant' and 'susceptible,' and 'avirulent' and 'virulent,' respectively). Genetically, resistance and avirulence are usually dominant (although dominance can be ignored in haploid pathogens). The resistant host is resistant to the avirulent pathogen, but susceptible to the virulent pathogen; the susceptible host, on the other hand, is susceptible to both pathogen phenotypes. Resistance involves a reduction in disease intensity that may be partial or complete. This host-pathogen interaction causes the different host and pathogen phenotypes to experience different fitnesses according to the phenotype of the other species with which they interact. In addition, it is necessary to assume that there are fitness costs associated with resistance and virulence alleles, otherwise the coevolutionary process simply terminates in the state in which all hosts are resistant and all pathogens are virulent.

The coevolutionary dynamics of host-pathogen systems are usually represented as a $2 \mathrm{D}$ phase diagram with the frequency of one host allele plotted against the frequency of one pathogen allele; we prefer to use the recessive allele in each species (Fig. 1). The long-term (equilibrium) behavior of a system may result in attraction to a stable equilibrium point, attraction to a stable limit cycle, neutral cycles, an unstable equilibrium point, or an unstable limit cycle.

Significant changes in the long-term behavior of the coevolutionary process are caused by almost trivial changes in the way that the selection process is modeled $(2,10,11)$. First, Leonard distinguished between 'hard' and 'soft' selection in the pathogen population. In hard selection, the cost of virulence is the same in infections on both host phenotypes, whereas, in soft selection, the cost of virulence only applies to infections of susceptible hosts. The hard selection version less often gives a stable polymorphism in both species than does the soft selection (10).

Second, Leonard distinguished between immediate reciprocal genetic feedback and delayed feedback between host and pathogen populations. In delayed feedback, the genetic changes due to selection in host and pathogen populations occur sequentially, whereas, in immediate feedback, the changes occur simultaneously (in parallel) (see below for mathematical detail). The de-

Corresponding author: J. J. Burdon; E-mail address: j.burdon@pi.csiro.au

Publication no. P-1997-0306-010

(C) 1997 The American Phytopathological Society layed feedback gives a stable equilibrium, whereas immediate feedback gives instability for the same fitness parameters.

\section{THE PROBLEMS IN LEONARD}

Leonard (10) used extensive computer simulations to study the equilibrium properties of his fitness model (9) for selection in a gene-for-gene interaction between host and pathogen populations. The stability properties of this model had remained unclear, despite investigations using various methods of analysis $(2,11,14)$. However, in clearing up some sources of controversy, Leonard (10) has generated results that are difficult to verify exactly. The most important result of his study was the discovery of unstable limit cycles, in which convergence to equilibrium allele frequencies did not occur when initial conditions lay outside a critical cycle. An example was given in Leonard (Fig. 2 in reference 10). Inside the cycle for Leonard's delayed feedback model of sequential gene frequency, changes in pathogen, and host populations, any starting point eventually went to the joint equilibrium frequencies in diminishing cycles.

We were alerted to problems with his concept of unstable cycles when we were unable to exactly replicate the results shown in Leonard's (10) Figure 2. Certainly, starting with initial frequency of virulence values $\left(n_{0}\right)$ greater than 0.2 gave convergence to the equilibrium, but so did some values less than 0.18 , which are outside Leonard's unstable limit cycle. Experimentation with the simulation program showed that rewriting the algorithm used to simulate the dynamics of the system, changing from double precision to single precision arithmetic, or, for some parameter values, changing from GWBASIC to QBASIC (Microsoft Corp., Redmond, WA) with the same program changed the location of the critical value of $n_{0}$. Hence, the observed position of the unstable cycle depends upon the arithmetic used in the simulation. Although we have found different values for the location of the unstable limit cycle, we have confirmed the phenomenon that Leonard described. What happens is that the corner of phase space where all of the host population is susceptible and all of the pathogen population is virulent acts analogously to a black hole: a host-pathogen system that passes close by is dragged toward it. Even if the system escapes back into another orbit around the equilibrium point, eventually it is dragged into the corner and is unable to escape.

Leonard plotted the minimum value of $n$ (the frequency of virulence at its lowest point in the limit cycle) as $-\log (n)$ in an attempt to measure coevolutionary "stability." Those plots show allele frequencies less than $10^{-30}$, which are numerically and biologically implausible. The numerical difficulties arise because even double precision arithmetic will round off $1-q$ as 1.0 when $q$ is less than $10^{-17}$, and other roundoff errors can creep in slowly as successive generations are iterated. Thus, this unstable cycle phenomenon may simply be an artifact of rounding errors. Leonard argued that the biological implausibility of such low allele fre- 
quencies in finite populations (e.g., in a haploid population of $10^{12}$, the smallest allele frequency is $10^{-12}$ ) renders the unstable region "inaccessible" and that, in the real world, the polymorphisms would always proceed to the equilibrium condition. This argument raises two problems that need further investigation. First, with such low allele frequencies, mutational processes cannot be ignored and, therefore, the effect of mutation should be tested. Second, the recognition that the real world involves finite populations implies that random drift processes (due to finite population size) must also be studied. In this letter, we show how the inclusion of mutation eliminates the unstable cycle phenomenon. In addition, some aspects of finite population behavior are reported.

\section{EQUILIBRIUM CONDITIONS}

Details of derivations and terminology follow Leonard (10). Briefly, selection coefficients in the pathogen population are $t=$ cost to the avirulent pathogen of resistance in the host, $k=$ cost of virulence in the pathogen, and $a=$ the parameter that distinguishes between hard selection $(a=0)$ and soft selection $(a=k)$. The value of $t$ reflects the amount of resistance shown by the resistant host genotype to the avirulent pathogen ( $t=1$ implies no reproduction by the avirulent pathogen). Selection coefficients in the host population are $s=$ cost of infection of susceptible hosts and $c$ $=$ cost of resistance. For a haploid pathogen (with frequencies $n$ and $1-n$ of virulence and avirulence, respectively) and a diploid host with random mating (with frequencies $p$ and $q$ of resistance and susceptibility alleles, respectively), the equilibrium phenotype frequencies for the general model are

$$
\begin{aligned}
& \text { avirulence }:(1-n)=(a s+c) /(a s+t s) \\
& \text { resistance: }\left(1-q^{2}\right)=k /(a+t)
\end{aligned}
$$

The location of the equilibrium point in the host-pathogen phase space is most easily understood by taking the hard selection version of the model

$$
\begin{aligned}
& \text { avirulence: }(1-n)=c / t s \\
& \text { resistance: }\left(1-q^{2}\right)=k / t
\end{aligned}
$$

That is, the equilibrium frequency of avirulence is the ratio of the cost of resistance to the product of the cost of avirulence and the cost of susceptibility. Similarly, the equilibrium frequency of the resistance phenotype is the ratio of the cost of virulence to the cost of avirulence. There are two important features of the equilibrium point: (i) the reciprocal interaction between host and pathogen produces an equilibrium frequency in one partner of the interaction that depends upon selection coefficients in the other and (ii) the smaller the costs of resistance and virulence (or the larger the costs of avirulence and susceptibility), the closer the equilibrium point is to the (1.0 virulence, 1.0 susceptibility) corner of the phase diagram (4). Thus, of the four corners of the phase diagram in which both species are monomorphic, the closest corner to the equilibrium point is (1.0 virulence, 1.0 susceptibility), and this is
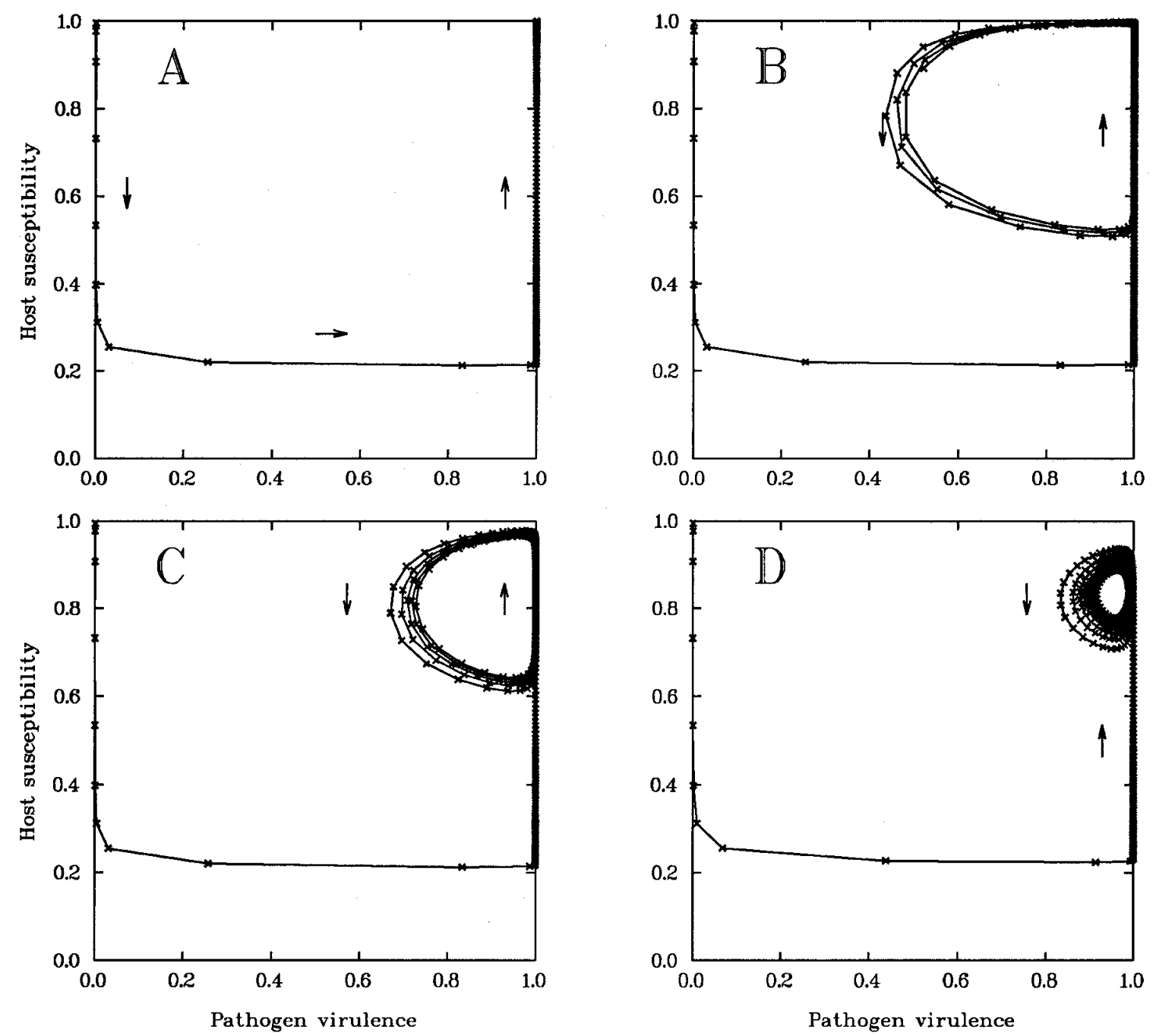

Fig. 1. The frequency of the susceptibility allele in the host population $(q)$ plotted against the frequency of the virulence allele in the pathogen population $(n)$ for 500 generations of Leonard's hard selection with delayed feedback $(t=1, s=0.8, k=0.3, c=0.03$, and $a=0$ [variables described in text]), starting from $(n=$ $0.001, q=0.999$ ) with mutation rates varying (for simplicity, forward and reverse rates are identical in both species). A, $\mu=0.0 . \mathbf{B}, \mu=10^{-10}$. C, $\mu=10^{-6} . \mathbf{D}, \mu=10^{-4}$. 
where "wandering" host-pathogen systems are most likely to end up. Thus, Leonard's (10) observation that smaller values of $c$ and $k$ gave less "stability" is explained by the fact that they produce orbits that must go closer to the corner that acts as a "black hole" because the equilibrium point is closer.

\section{MUTATION}

Genetic analysis of host resistance/susceptibility genes has shown that mutations from resistance to susceptibility are common, but mutations from susceptibility to resistance are not known from ordinary experiments (13). Since it is widely envisaged that dominant resistance alleles have a positive function and the recessive susceptibility alleles have lost that function, it is easy to imagine that many different mutations may produce susceptibility, but only one specific mutation may restore the resistance specificity. Detailed genetic studies of mutation and recombination at particular resistance loci have indicated that many such loci have considerable structural complexity and many mutationlike events are recombinational in origin. At the Rpl locus in maize, homozygotes for resistance alleles can produce susceptible progeny in test crosses at rates around $10^{-3}$ (varying between different alleles) by unequal crossing over events. Heterozygotes for two resistance specificities in trans produce recombinants with both or neither specificity $(6,7)$. In the latter case, for one specificity involved in one gene-for-gene interaction with a pathogen, the recombinants without either specificity would appear as sus-
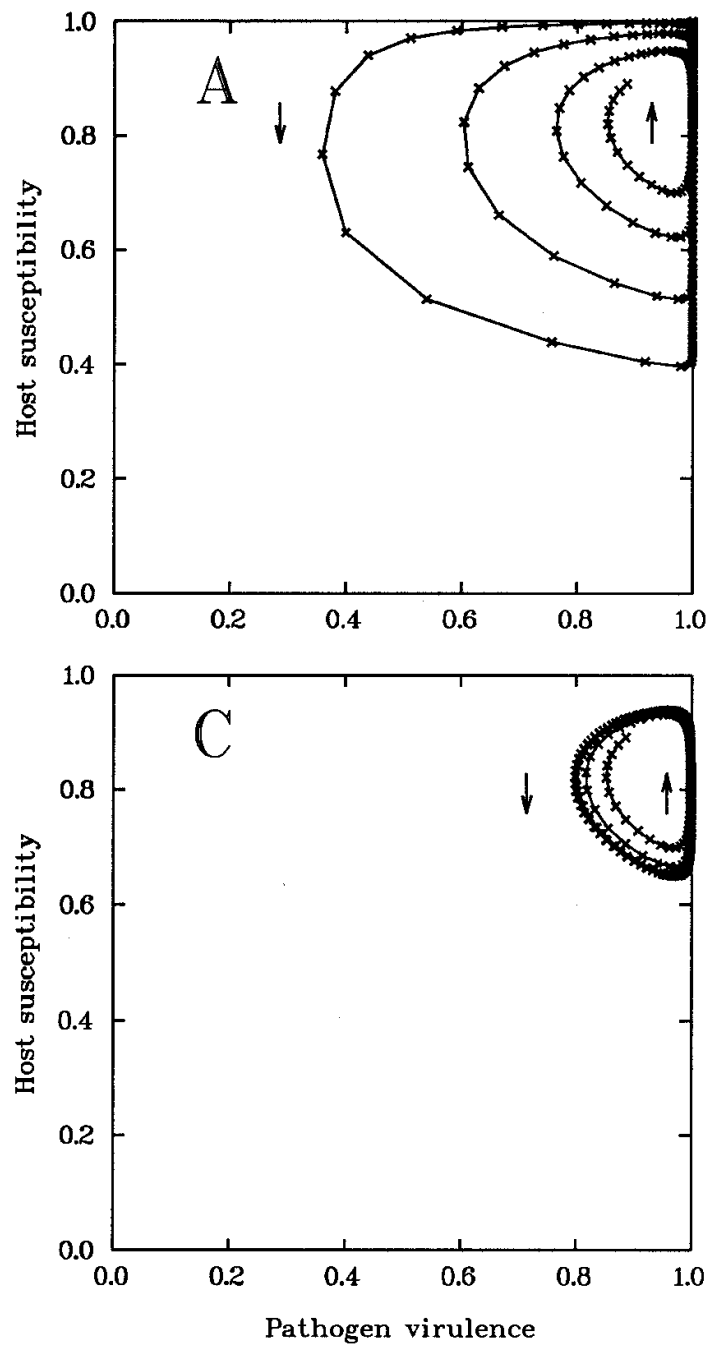

ceptibility "mutations" in very high frequency. The L locus in flax does not show high frequencies of susceptible mutants from homozygotes for resistance specificities, but heterozygotes for different specificities in trans can produce susceptible progeny at rates as high as nearly $10^{-3}(8)$. When some of these susceptible progeny were screened for reversion, revertants to certain specificities were obtained. Again, these genetic changes, which are probably due to recombination, will appear as high frequency mutations from the population genetics' point of view.

Thus, there appears to be good experimental evidence that, for at least some complex loci involved in gene-for-gene interactions, "mutation" rates for single resistance specificities may be very high in both directions, because of recombination/unequal crossing over events. Indeed, this process may well explain the origin of such loci. In other cases in which frequencies of forward and back mutation rates may be very different and both of very low magnitudes $\left(10^{-6}\right.$ to $10^{-10}$ ), more usual mutational mechanisms (base changes and insertion/deletion events) provide an adequate explanation.

For pathogens, mutations from avirulence to virulence are easier to study than the reciprocal. However, not many systems have been studied, and the estimates vary widely from high $\left(\sim 10^{-5}\right)$ to low $\left(\sim 10^{-8}\right)$ (1) with different loci in the same species mutating at different rates $(3,12,15)$. Mutation from virulence to avirulence is difficult to study; therefore, there are no estimates of mutation rates in that direction.

The obvious conclusion from this brief review of mutation in gene-for-gene systems is that a range of mutation rates has been ob-
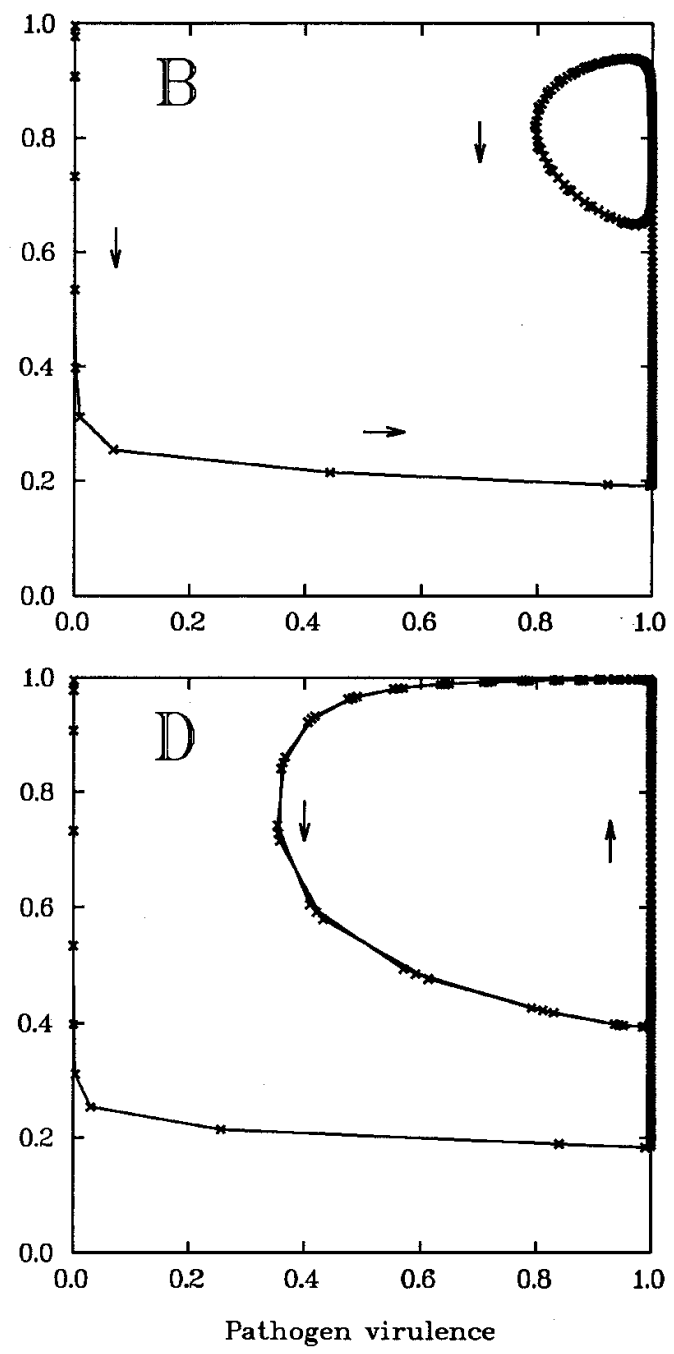

Fig. 2. Five hundred generations of reciprocal feedback model with variation in mutation rate and initial conditions. A, No mutation, starting from (0.9,0.9). B, $\mu=10^{-4}$, starting from (0.001,0.999). C, $\mu=10^{-4}$, starting from $(0.9,0.9)$. $\mathbf{D}, \mu=10^{-10}$, starting from $(0.001,0.999)$. 
served, and even the lowest rates are high enough to prevent allele frequencies in natural populations declining to infinitesimally small magnitudes. Thus, a choice of mutation rates from $10^{-4}$ to $10^{-10}$ should cover the likely range of values in natural populations. For simplicity, the differences in forward and back mutation rates will be ignored, and both host and pathogen populations will be given the same mutation rate. We will show how variations in the rate of mutation affects the behavior of Leonard's model of selection in a gene-for-gene system.

The effect of mutation at a rate, $\mu$, in each direction in both species is computed as follows:

$$
\begin{aligned}
& n^{\prime}=n+\mu(1-2 n) \\
& q^{\prime}=q+\mu(1-2 q)
\end{aligned}
$$

in which $n$ and $q$ are the frequency of virulence and susceptibility before mutation, and $n^{\prime}$ and $q^{\prime}$ are their values after mutation occurs. From inspection of these equations, it can be seen that the allele frequencies in each species are now restricted to the range $\mu$ to $1-\mu$, so very tiny frequencies are no longer possible.

\section{FINITE POPULATIONS}

An additional process occurring in finite populations is random genetic drift, in which the expected gene frequency changes in each generation will vary randomly by an amount inversely pro- portional to population size. In a large population, the effect of random drift will be negligible unless gene frequencies are close to 0 or 1 , when they may, by chance, drift to 0 or 1 . In small populations, random drift is much more important and can lead to loss of polymorphism in gene-for-gene systems, especially when selection takes gene frequencies close to 0 or 1 . For simplification, both populations will be given the same size $(N)$, but because the host is diploid with $2 N$ genes at each locus, the pathogen population with only $N$ genes will have more random drift than the host population.

\section{POPULATION SUBDIVISION}

We view population structure, based on the subdivision of host and pathogen populations into separate patches, colonies, or subpopulations within a metapopulation, as potentially contributing significantly to coevolution between hosts and pathogens. Hence, in our finite population model, we use Wright's (17) island model of gene flow to compare two scenarios: (i) a single large population of 10,000 and (ii) a subdivided population of 100 colonies each containing 100 individuals, in which all colonies exchange genes at random with all other colonies, regardless of geographic proximity. Standard theory for island models with neutral genes shows that the interaction between gene flow and random drift within colonies changes when $m$ (the proportion of each colony
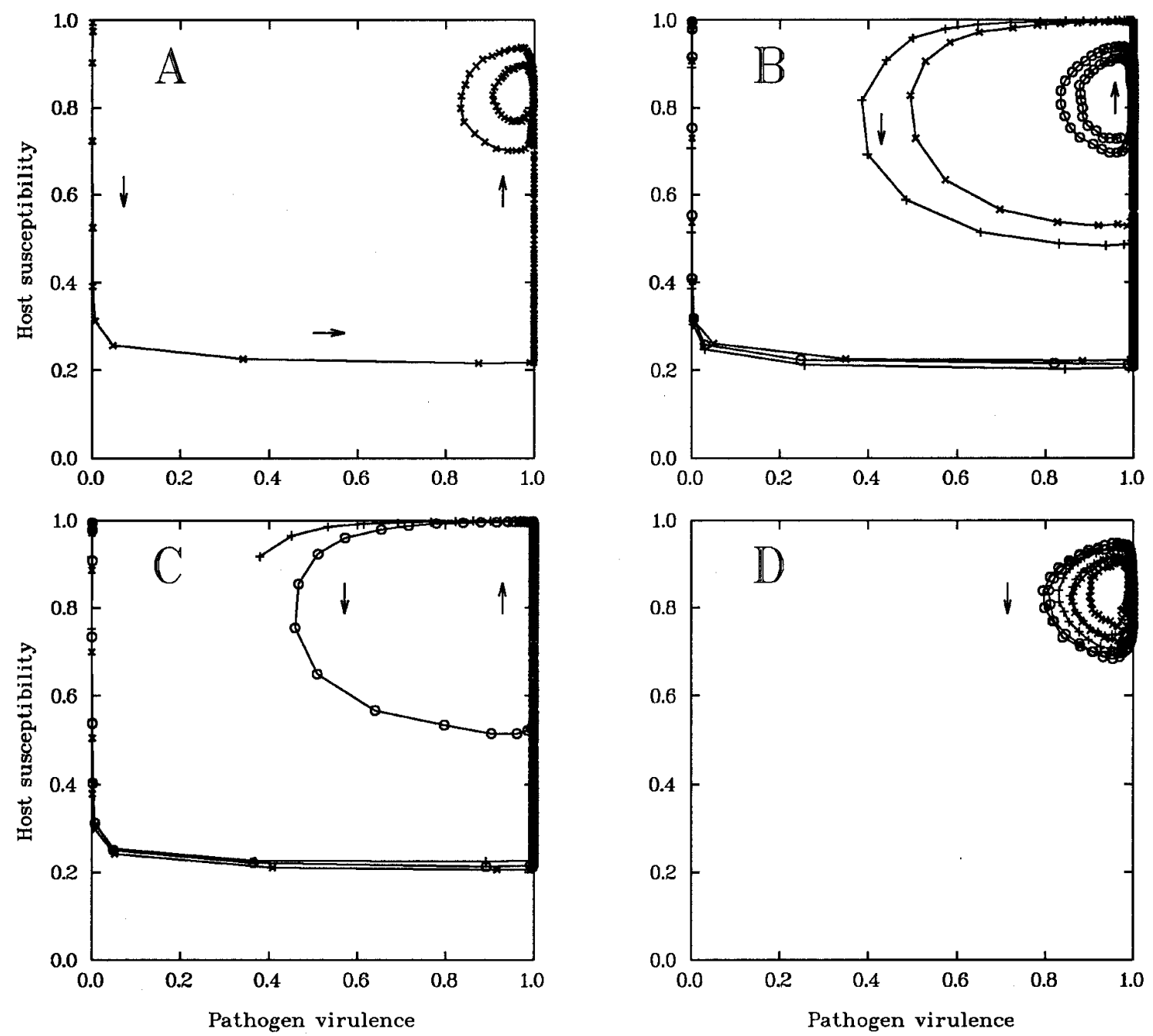

Fig. 3. Random drift and selection in a population of 10,000 diploid hosts and 10,000 haploid pathogens. A, One replicate of $\mu=10^{-4}$ starting from $(0.001,0.999)$ for 200 generations (other replicates almost identical). B, Three replicates of $\mu=10^{-5}$ starting from $(0.001,0.999)$ for 200 generations. C, Three replicates of $\mu=10^{-6}$ starting from $(0.001,0.999)$ for 200 generations. D, Three replicates of $\mu=10^{-5}$ starting from $(0.9625,0.83667)$ (the equilibrium point for the selection parameters) for generations 400 to 500 . 
that is immigrant in each generation) is about $1 / N$ (in which $N$ is the colony size) (17). For gene flow between colonies, high $(m=$ $4 / N)$, medium $(m=1 / N)$, and low $(m=1 / 4 N)$ rates were used.

\section{RESULTS AND DISCUSSION}

To illustrate our results, the fitnesses used in Leonard's (10) Figure 2 were chosen $(a=0, t=1, s=0.8, c=0.03$, and $k=0.3)$, and a starting point of $n_{0}=0.001$ and $q_{0}=0.999$ was used, because it is well outside Leonard's unstable limit cycle. Furthermore, it seems sensible to expect that a system that begins with a largely susceptible host population and largely avirulent pathogen population should go to equilibrium. Figure 1 shows the outcome of 500 generations with four different levels of mutation. When no mutation occurs (Fig. 1A), the interaction is attracted to the $(1,1)$ corner, in which a susceptible host population is attacked by a virulent pathogen population. Even a small mutation rate $\left(10^{-10}\right)$ (Fig. 1B) allows the system to escape the corner and execute three converging cycles in 500 generations. Higher mutation rates (Fig. $1 \mathrm{C}$ and D) produce smaller and more rapid cycles with convergence towards the equilibrium point. Thus, the addition of even a small amount of reciprocal mutation to the model abolishes the unstable limit cycle behavior.

These calculations were performed with delayed feedback $\left(n_{i+1}\right.$ $=g\left(q_{i}, n_{i}\right)$ and $q_{i+1}=f\left(q_{i}, n_{i+1}\right)\left[g\left(q_{i}, n_{i}\right)\right.$ and $f\left(q_{i}, n_{i+1}\right)$ are functions of $p_{i}$ and $n_{i}$, the frequencies of the appropriate genes in the $i$ th generation]). Delayed feedback results in gene frequency changes in the host population that are driven by the new gene frequencies after selection has acted on the pathogen population. This model allows stable equilibria. In contrast, the reciprocal genetic feedback model has the same calculation for the pathogen, but the host is affected by the initial allele frequencies in the pathogen population $\left(q_{i+1}=f\left(q_{i}, n_{i}\right)\right)$. In this case, the equilibrium is not stable. The effect of mutation on the outcome of the reciprocal feedback model was examined by taking the same selection model and mutation rates as above. From all starting points tried, in the absence of mutation, the system went to fixation $(1.0,1.0)$ (Fig. 2A shows the result of starting from $(0.9,0.9))$. The introduction of mutation produced limit cycle behavior. For the highest mutation rate, Figure 2B shows approach to the cycle from $(0.001,0.999)$, and Figure $2 \mathrm{C}$ shows the approach from $(0.9,0.9)$. The same cycle is produced in each case, and, since the cycle is approached from both directions, it is stable. A low mutation rate $\left(10^{-10}\right)$ produces a limit cycle with larger amplitude (Fig. 2D). An interesting feature of these simulations is the speed with which convergence to the limit cycle occurs. Subsequent simulations use the delayed feedback model.

The results of the interaction of finite populations of 10,000 hosts and pathogens with no mutation was simple. When starting from $(0.001,0.999)$, most simulations ended with fixation of viru-
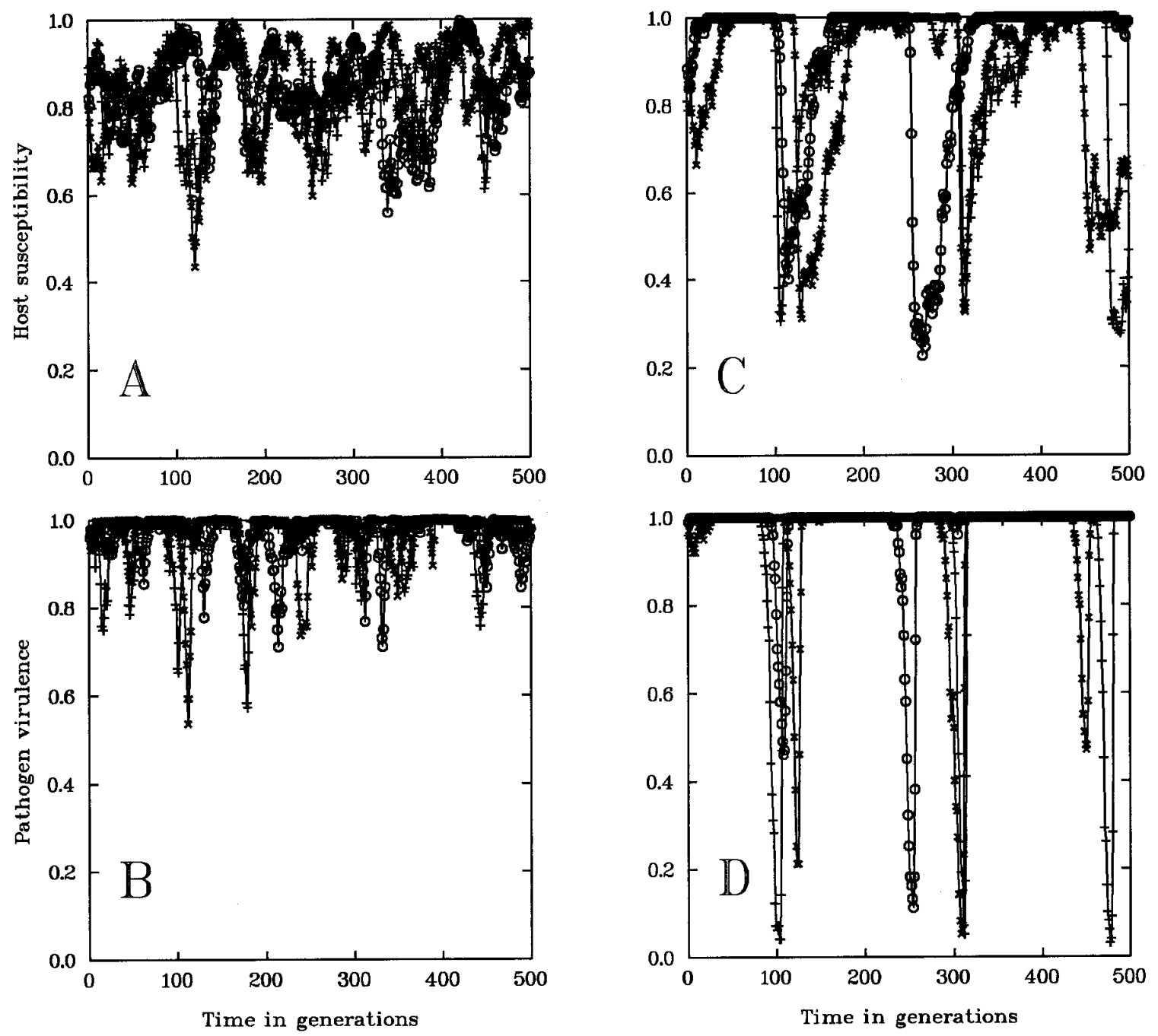

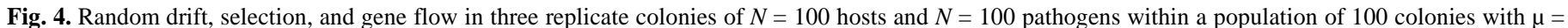

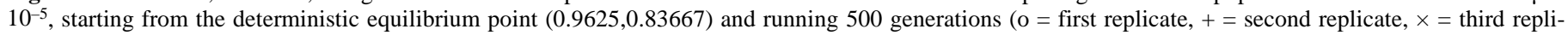

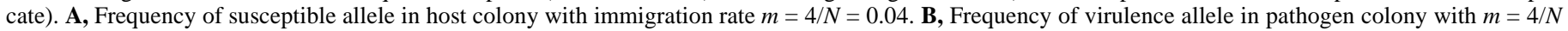
$=0.04$. C, Susceptible allele frequency with $m=1 / 4 N=0.0025$. D, Virulence allele frequency with $m=1 / 4 N=0.0025$. 
lence and susceptibility and only a few went to fixation of avirulence and resistance. Loss or fixation of virulence was rapid, usually within 20 generations. The introduction of mutation at high rates prevented fixation and, at lower rates, could restart a cycle that had been trapped in a corner of the phase diagram. Figure $3 \mathrm{~A}$ shows 200 generations of one of three replicates with high mutation rate $\left(10^{-4}\right)$; the behavior of all replicates is similar to the deterministic case (Fig. 1D). With slightly lower mutation rate $\left(10^{-5}\right)$, the three replicates (Fig. 3B) are more variable with two achieving only one large cycle and one managing two small cycles in 200 generations. A lower mutation rate $\left(10^{-6}\right)$ gives three very different replicates: after 200 generations, one remained stalled in the $(1.0,1.0)$ corner; one had just escaped and started a cycle; and the third had completed a cycle (Fig. 3C). These results suggest that it is the magnitude of $N \mu$ (the expected number of mutations per generation in the population) that determines behavior. When $N \mu<<1$, the population can become trapped in the $(1,1)$ corner until a mutation occurs that will "kick" it out of the corner. When $N \mu>1$, the population will not be trapped in a corner.

A feature of the finite populations is that they do not remain at the stable equilibrium point. Random drift leads to oscillations about the equilibrium frequency in both species, which drives the system into irregular cycles. The results of three replicates for generations 400 to 500 , after starting from the selection equilibrium point, are shown in Figure 3D. These cycles vary in amplitude, so they cannot be described as stable, yet the cyclic behavior is consistent and reproducible.

In the subdivided population, gene flow rates affect the cyclical dynamics of the whole metapopulation, as well as that of individual colonies. With high gene flow $(m=4 / N)$, the whole population behaves much the same as for a single population of 10,000 , and the individual colonies also have irregular cycles that are only roughly synchronized between different colonies (Fig. 4A and B). Most of the time, host colonies remain polymorphic for resistance/susceptibility, whereas pathogen colonies are often fixed for virulence. With low gene flow $(m=1 / 4 N)$, the cyclical dynamics are slowed down both in the metapopulation and individual colonies. Within colonies, the increased wavelength of the cycles is accompanied by increased amplitude (Fig. 4C and D), so that, apart from brief periods of polymorphism, host colonies are often fully susceptible and pathogen colonies are usually fixed for virulence.

In summary, adding reverse mutation at biologically reasonable frequencies causes important changes to the properties of Leonard's host-pathogen models. Unstable limit cycles disappear and, in the unstable immediate feedback model, the system goes to stable limit cycles. Stable equilibria in the delayed feedback model become irregular cycles in finite populations.

These results suggest that the essential difference between the dynamics of Leonard's original model and the dynamics observed in Frank's (4) ecologically orientated model based on LotkaVolterra equations is caused by Leonard's neglect of mutation. Hamilton (5), in a host with matching parasites model, also showed that inclusion of mutation reduced the likelihood of genetic fixation. All three models show that mutation has an important role to play in maintaining polymorphism under conditions in which gene fixation would otherwise occur.

\section{ACKNOWLEDGMENT}

Part of this work was carried out while G. C. Kirby was a visiting scientist in the CSIRO Division of Plant Industry.

\section{LITERATURE CITED}

1. Burdon, J. J. 1992. Genetic variation in pathogen populations and its implications for adaptation to host resistance. Pages 41-56 in: Durability of Disease Resistance. Th. Jacobs and J. E. Parlevliet, eds. Kluwer Academic Publishers, Amsterdam.

2. Fleming, R. A. 1980. Selection pressures and plant pathogens: Robustness of the model. Phytopathology 70:175-178

3. Flor, H. H. 1958. Mutations to wider virulence in Melampsora lini. Phytopathology 48:297-301.

4. Frank, S. A. 1993. Coevolutionary genetics of plants and pathogens. Evol. Ecol. 7:45-75.

5. Hamilton, W. D. 1993. Haploid dynamic polymorphism in a host with matching parasites: Effects of mutation/subdivision, linkage and patterns of selection. J. Hered. 84:328-338.

6. Hu, G., and Hulbert, S. 1994. Evidence for the involvement of gene conversion in meiotic instability of the $R p 1$ rust resistance genes of maize. Genome 37:742-746.

7. Hulbert, S. H., Sudupak, M. A., and Hong, K. S. 1993. Genetic relationships between alleles of the Rpl rust resistance locus of maize. Mol. Plant-Microbe Interact. 6:387-392.

8. Islam, M. R., Shepherd, K. W., and Mayo, G. M. E. 1989. Recombination among genes at the $\mathrm{L}$ group in flax conferring resistance to rust. Theor. Appl. Genet. 77:540-546.

9. Leonard, K. J. 1977. Selection pressures and plant pathogens. Ann. N.Y. Acad. Sci. 287:207-222.

10. Leonard, K. J. 1994. Stability of equilibria in a gene-for-gene coevolution model of host-parasite interactions. Phytopathology 84:70-77.

11. Leonard, K. J., and Czochor, R. J. 1978. In response to 'Selection pressures and plant pathogens: Stability of equilibria.' Phytopathology 68:971-973.

12. Luig, N. H. 1978. Mutation studies in Puccinia graminis tritici. Pages 533-539 in: Proc. Int. Wheat Genet. Symp., 5th. Ind. Soc. Genet. Plant Breed., New Delhi.

13. Pryor, A., and Ellis, J. G. 1993. The genetic complexity of fungal resistance genes in plants. Adv. Plant Pathol. 10:281-305.

14. Sedcole, J. R. 1978. Selection pressures and plant pathogens: Stability of equilibria. Phytopathology 68:967-970.

15. Statler, G. D. 1990. New mutations from a mutant culture of Puccinia recondita. Can. J. Plant Pathol. 12:243-246.

16. Thompson, J. N., and Burdon, J. J. 1992. Gene-for-gene coevolution between plants and parasites. Nature 360:121-125.

17. Wright, S. 1969. Evolution and the Genetics of Populations. Vol. 2: The Theory of Gene Frequencies. Chicago University Press, Chicago. 